\title{
The Influence of Additive Allee Effect and Periodic Harvesting to the Dynamics of Leslie-Gower Predator- Prey Model
}

\author{
Hasan S. Panigoro' ${ }^{*}$, Emli Rahmi ${ }^{2}$, Novianita Achmad ${ }^{3}$, Sri Lestari Mahmud ${ }^{4}$ \\ 1,2,3,4 Department of Mathematics, Faculty of Mathematics and Natural Sciences, \\ Universitas Negeri Gorontalo \\ Jl. Jenderal Sudirman No. 6, Kota Gorontalo 96128, Gorontalo, Indonesia \\ *Corresponding Author.Email: hspanigoro@ung.ac.id
}

\begin{abstract}
In this paper, the influence of additive Allee effect in prey and periodic harvesting in predator to the dynamics of the Leslie-Gower predator-prey model is proposed. We first simplify the model to the nondimensional system by scaling the variable and transform the model into an autonomous system. If the effect Allee is weak, we have at most two equilibrium points, else if the Allee effect is strong, at most four equilibrium points may exist. Furthermore, the behavior of the system around equilibrium points is investigated. In the end, we give numerical simulations to support theoretical results.

Keywords:

Leslie-Gower; Allee Effect; Periodic Harvesting; Non-autonomous

Citation Format:

H. S. Panigoro, E. Rahmi, N. Achmad, and S. L. Mahmud, "The Influence of Additive Allee Effect and Periodic Harvesting to the Dynamics of Leslie-Gower Predator-Prey Model," Jambura J. Math., vol. 2, no. 2, pp.87-96, 2020
\end{abstract}

\section{Introduction}

In the past few years, the predator-prey model is studied intensively due to its natural capability to describes the existence and extinction of the population caused by the interaction between prey and its predator [1]. Several references show that the predatorprey model is modified to harmonize with an actual condition such as prey refuge [2][3] and infectious diseases in the population [4][5]. The popular one is the famous LeslieGower predator-prey model [6] which is defined as follows.

$$
\begin{aligned}
& \frac{d x}{d t}=r x\left(1-\frac{x}{K}\right)-p x y, \\
& \frac{d y}{d t}=s y\left(1-\frac{y}{n x}\right),
\end{aligned}
$$

where $x(t)>0$ and $y(t) \geq 0$ are the density of prey and predator, respectively. Both populations are growth logistically where the carrying capacity of predator depends on the density of predator. For further, the biological interpretation of parameters is defined in Table 1. 


\section{Panigoro et.al}

One interesting phenomenon in ecological modeling is the Allee effect, which represents the decrease in per capita fertility rate as a result of several mechanisms such as lowdensity population, intraspecific competition, and difficulties in finding mates [7][8]. Several researchers using different definitions to represents the Allee effect in a predatorprey model such as multiplicative Allee effect [9][10], additive Allee effect [11]-[13], and double Allee effect [14][15]. Particularly, we are interested to study the Allee effect deduced in [16]-[19] which consider the natural growth function given by

$$
\frac{d x}{d t}=r x\left(1-\frac{x}{K}-\frac{m x}{x+b}\right)
$$

which called additive allee effect. The density of a population is decreased which is shown by the presence of the Allee effect function $f(x)=\frac{m x}{x+b}$ in the logistic growth model. We applied the additive Allee effect to the prey in the system (1) and obtain the following system.

$$
\begin{aligned}
& \frac{d x}{d t}=\left[r\left(1-\frac{x}{K}\right)-\frac{m}{x+b}\right] x-p x y, \\
& \frac{d y}{d t}=s y\left(1-\frac{y}{n x}\right),
\end{aligned}
$$

where the Allee parameters $m$ and $b$ have the following biological interpretation.

1. The weak Allee effect if $0<m<b$, and

2. The strong Allee effect if $m>b$.

Table 1. Biological interpretation of system (1)

\begin{tabular}{cl}
\hline Parameters & \multicolumn{1}{c}{ Biological interpretation } \\
\hline$r$ & The intrinsic growth rate of prey \\
$s$ & The intrinsic growth rate of predator \\
$K$ & The environmental carrying capacity of prey \\
$p$ & The maximal predator per capita consumption \\
$n$ & The measure of the quality of food \\
\hline
\end{tabular}

Source: [11][20]

Apart from the Allee effect, one that caused a decrease in population density is the exploitation of the population through harvesting. In some conditions, the harvesting is done periodically due to seasonal activities such as hunting season, migration of population, weather, and so on. This periodically harvesting means that the harvesting is still exists as $t \rightarrow \infty$, but the harvesting rate changes periodically and also proportional to the density of predator. We replace the conventional proportional effort harvesting $H(y)=h_{y} y$ with a harvesting periodic function $H(y)=(1+\varepsilon \sin (w t)) h_{y} y$. By using this assumption, the system (3) is transformed into a non-autonomous system as follows.

$$
\begin{aligned}
& \frac{d x}{d t}=\left[r\left(1-\frac{x}{K}\right)-\frac{m}{x+b}\right] x-p x y, \\
& \frac{d y}{d t}=s y\left(1-\frac{y}{n x}\right)-(1+\varepsilon \sin (w t)) h_{y} y,
\end{aligned}
$$


where $h_{y}$ is harvesting parameter, $0<\varepsilon<1$ is harvesting constant, and $w$ is the period parameter. The harvesting function is periodically changed in $(0, h]$. Studying the dynamics of the system (4) is the main topic in our works.

This paper is organized as follows: In Section 2, we give the methods used in our research. In Section 3, all of our research results are presented and discussed. Finally, the conclusion of our research is given in Section 4 .

\section{Methods}

The dynamics of the system (4) is investigated and presented using several methods as follows.

1. Transforming system (4) to an autonomous non-dimensional system by scaling the variable and expanding the dimension of system.

2. Identifying the existence of equilibrium points and their local stability of the autonomous system for both strong and weak Allee effect.

3. Plotting the numerical solutions of the model to support the theoretical results by using $4^{\text {th }}$-order Runge-Kutta schemes.

\section{Results and Discussion}

\subsection{Non-dimensional System}

We first simplify system (4) to a non-autonomous non-dimensional system by applying variable scaling $(x, y, t) \rightarrow(x / K, y / n K, r t)$ and obtain

$$
\begin{aligned}
& \frac{d x}{d t}=\left[1-x-\frac{\alpha}{x+\beta}-\kappa y\right] x, \\
& \frac{d y}{d t}=\left[\delta\left(1-\frac{y}{x}\right)-(1+\varepsilon \sin (\omega t)) h\right] y,
\end{aligned}
$$

where $\alpha=m / r K, \eta=b / K, \delta=s / r, \kappa=n p K / r, \omega=w / r$ and $h=h_{y} / r$. These methods guarantee that the number of parameters is reduced and the dynamics of system (5) are qualitatively similar to the system (4). We can easily check that the prey has a weak Allee effect if $0<\alpha<\beta$ and strong Allee effect if $\alpha>\beta$.

\subsection{Autonomous System}

Although system (5) is simpler than system (4), the dynamics of the system (5) is still difficult to investigate due to its dependency on time $t$ explicitly which is called a nonautonomous system. In this part, we show the way to transform the non-autonomous system (5) to an autonomous system. Suppose that

$$
\begin{aligned}
& \frac{d u}{d t}=-u+\omega v+u\left(u^{2}+v^{2}\right), \\
& \frac{d v}{d t}=-\omega u-v+v\left(u^{2}+v^{2}\right) .
\end{aligned}
$$

It can easily prove that the system (6) has solution $u(t)=\sin (\omega t)$ and $v(t)=\cos (\omega t)$. By utilizing the system (6), we have an autonomous form of system (5) as follows. 


$$
\begin{aligned}
& \frac{d x}{d t}=\left[1-x-\frac{\alpha}{x+\beta}-\kappa y\right] x, \\
& \frac{d y}{d t}=\left[\delta\left(1-\frac{y}{x}\right)-(1+\varepsilon u) h\right] y, \\
& \frac{d u}{d t}=-u+\omega v+u\left(u^{2}+v^{2}\right), \\
& \frac{d v}{d t}=-\omega u-v+v\left(u^{2}+v^{2}\right) .
\end{aligned}
$$

From the biological point of view, the solution of system (7) must satisfying

$$
\mathbb{R}_{+}^{4}:=\{(x, y, u, v): x>0, y \geq 0, x, y, u, v \in \mathbb{R}\} .
$$

\subsection{Existence of Equilibrium Point}

In this part, we obtain the equilibrium point of system (7) by solving

$$
\begin{aligned}
{\left[1-x-\frac{\alpha}{x+\beta}-\kappa y\right] x } & =0 \\
{\left[\delta\left(1-\frac{y}{x}\right)-(1+\varepsilon u) h\right] y } & =0 \\
-u+\omega v+u\left(u^{2}+v^{2}\right) & =0 \\
-\omega u-v+v\left(u^{2}+v^{2}\right) & =0 .
\end{aligned}
$$

Notice that $u(t)$ and $v(t)$ are fulfilled eq. $(8)$ if $(u, v)=(0,0)$ or $u^{2}+v^{2}=1$. In our work, we focus on studying the dynamics when $(u, v)=(0,0)$. Therefore, we obtain the following equilibrium points.

1. The predator extinction points $\hat{E}_{1,2}=\left(\hat{x}_{1,2}, 0,0,0\right)$ where $\hat{x}_{1,2}$ are solutions of quadratic polynomial

$$
x^{2}-(1-\beta) x+\alpha-\beta=0 .
$$

By solving eq. (9), we obtain

$$
\hat{x}_{1}=\frac{1-\beta}{2}+\sqrt{\left(\frac{\beta+1}{2}\right)^{2}-\alpha}, \quad \hat{x}_{2}=\frac{1-\beta}{2}-\sqrt{\left(\frac{\beta+1}{2}\right)^{2}-\alpha} .
$$

Notice that both equilibrium points may exist if $\alpha<\left(\frac{\beta+1}{2}\right)^{2}$. When the Allee effect is weak, the predator extinction point $\hat{E}_{1}$ is the only possible equilibrium point that appears. When the Allee effect is strong, both predator extinction point $\hat{E}_{1,2}$ are exist if $0<\beta<1$.

2. The co-existence point $E_{1,2}^{*}=\left(x_{1,2}^{*},\left(1-\frac{h}{\delta}\right) x_{1,2}^{*}, 0,0\right)$ where $x_{1,2}^{*}$ are solutions of quadratic polynomial

$$
x^{2}-\xi_{1} x+\xi_{2}=0, \quad \xi_{1}=\beta-\frac{\delta}{\delta+(\delta-h) \kappa}, \quad \xi_{2}=\frac{(\alpha-\beta) \delta}{\delta+(\delta-h) \kappa} .
$$

Thus, we have 


$$
x_{1}^{*}=-\frac{1}{2}\left(\xi_{1}-\sqrt{\xi_{1}^{2}-4 \xi_{2}}\right), \quad x_{2}^{*}=-\frac{1}{2}\left(\xi_{1}+\sqrt{\xi_{1}^{2}-4 \xi_{2}}\right) .
$$

Both equilibrium points are exist if $h<\delta$ and $x_{1,2}^{*}>0$. Making use eq. (12), we have $x_{1,2}^{*} \in \mathbb{R}$ if $\xi_{1}^{2}>4 \xi_{2}$. When the Allee effect is weak, $E_{1}^{*}$ is the only equilibrium point in interior. If the Allee effect is strong, $E_{1,2}^{*}$ are exist in interior of system (7).

Therefore, we have at most two equilibrium points for weak Allee effect and four equilibrium points for a strong Allee effect. Next, the local stability of those equilibrium points is investigated.

\subsection{Stability of Equilibrium Point}

In this part, we present the local stability of equilibrium point both strong and weak Allee effect. To investigate the dynamics around an equilibrium point, we identify the Jacobian matrix as follows.

where

$$
J(x, y, u, v)=\left[\begin{array}{cccc}
\eta_{1} & -\kappa x & 0 & 0 \\
\frac{\delta y^{2}}{x^{2}} & \eta_{2} & -\varepsilon h y & 0 \\
0 & 0 & \eta_{3} & \eta_{4} \\
0 & 0 & \eta_{5} & \eta_{6}
\end{array}\right],
$$

$$
\begin{array}{lll}
\eta_{1}=1-2 x-\frac{\alpha}{x+\beta}+\frac{\alpha x}{(x+\beta)^{2}}-\kappa y, & \eta_{4}=2 u v+\omega \\
\eta_{2}=\delta\left(1-\frac{y}{x}\right)-\frac{\delta y}{x}-(\varepsilon u+1) h, & \eta_{5}=2 u v-\omega \\
\eta_{3}=3 u^{2}+v^{2}-1, & \eta_{6}=u^{2}+3 v^{2}-1 .
\end{array}
$$

Theorem 1. The predator extinction point $\hat{E}_{1}$ is locally asymptotically stable if $h>\delta$.

Proof. By replacing $(x, y, u, v)$ in eq. (13) with $\hat{E}_{1}$, the Jacobian matrix (13) becomes

$$
J\left(\hat{E}_{1}\right)=\left[\begin{array}{cccc}
\frac{\alpha \hat{x}_{1}}{\left(\hat{x}_{1}+\beta\right)^{2}}-\hat{x}_{1} & -\kappa \hat{x}_{1} & 0 & 0 \\
0 & \delta-h & 0 & 0 \\
0 & 0 & -1 & \omega \\
0 & 0 & -\omega & -1
\end{array}\right],
$$

and gives the eigenvalues as follows: $\lambda_{1}=\frac{\alpha \hat{x}_{1}}{\left(\hat{x}_{1}+\beta\right)^{2}}-\hat{x}_{1}, \lambda_{2}=\delta-h$, and $\lambda_{3,4}=-1 \pm \omega i$. It is easily confirmed that $\lambda_{2}<0$ when $h>\delta$. It is also clear that $\operatorname{Re}\left(\lambda_{3,4}\right)<0$. Thus, the stability of $\hat{E}_{1}$ depend on the sign of $\lambda_{1}$. By substituting the value of $\hat{x}_{1}$ and making use of the existence condition of $\hat{E}_{1}$, the sign of $\lambda_{1}$ is definitely negative. Furthermore, $\hat{E}_{1}$ is locally asymptotically stable and Theorem 1 is completely proven.

Remark. We have shown that if $\hat{E}_{1}$ is locally asymptotically stable then $E_{1,2}^{*}$ are not exists.

Theorem 2. The predator extinction point $\hat{E}_{2}$ is always a saddle point.

Proof. System (7) has a quite similar Jacobian matrix to eq. (15). Thus we have eigenvalues $\lambda_{1}=\frac{\alpha \hat{x}_{2}}{\left(\hat{x}_{2}+\beta\right)^{2}}-\hat{x}_{2}, \lambda_{2}=\delta-h$, and $\lambda_{3,4}=-1 \pm \omega i$. Therefore, the stability of 


\section{Panigoro et.al}

$\widehat{E}_{2}$ depend on $\lambda_{1}$. By considering the existence condition and strong Allee effect condition, we obtain that $\lambda_{1}$ is always positive. Thus, we always at most one positive eigenvalue and two eigenvalue satisfying $\operatorname{Re}(\lambda)<0$ and hence $\hat{E}_{2}$ is always a saddle point.

Theorem 3. Suppose that

$$
\begin{aligned}
\varphi_{1,2} & =\left(\frac{\alpha}{\left(x_{1,2}^{*}+\beta\right)^{2}}-1\right) x_{1,2}^{*}-\left(\kappa x_{1,2}^{*}+\delta\right)\left(1-\frac{h}{\delta}\right) \\
\phi_{1,2} & =\left[2\left(1-\frac{h}{\delta}\right) \kappa-\frac{\alpha}{\left(x_{1,2}^{*}+\beta\right)^{2}}+1\right]\left(1-\frac{h}{\delta}\right) \delta x_{1,2}^{*}
\end{aligned}
$$

The co-existence point $E_{1}^{*}$ and $E_{2}^{*}$ are locally asymptotically stable if $\varphi_{1}<0$ and $\phi_{1}>0$; and $\varphi_{2}<0$ and $\phi_{2}>0$, respectively.

Proof. Firstly. We identify the stability of $E_{1}^{*}$. By substituting $E_{1}^{*}$ to eq. (13), we achieve

$$
J\left(\hat{E}_{1}\right)=\left[\begin{array}{cccc}
\frac{\alpha x_{1}^{*}}{\left(x_{1}^{*}+\beta\right)^{2}}-\kappa\left(1-\frac{h}{\delta}\right) x_{1}^{*}-x_{1}^{*} & -\kappa x_{1}^{*} & 0 & 0 \\
\delta\left(1-\frac{h}{\delta}\right)^{2} & -\delta\left(1-\frac{h}{\delta}\right) & -\varepsilon h\left(1-\frac{h}{\delta}\right) x_{1}^{*} & 0 \\
0 & 0 & -1 & \omega \\
0 & 0 & -\omega & -1
\end{array}\right] .
$$

Therefore, we have the eigenvalues as follows:

$$
\begin{aligned}
& \lambda_{1,2}=\frac{1}{2}\left(\varphi_{1} \pm \sqrt{\varphi_{1}^{2}-4 \phi_{1}}\right) \\
& \lambda_{3,4}=-1 \pm \omega i
\end{aligned}
$$

Thus, according to the trace-determinant theorem, $E_{1}^{*}$ is locally asymptotically stable if $\varphi_{1}<0$ and $\phi_{1}>0$. Using the similar manner, the stability condition of $E_{2}^{*}$ is also proven.

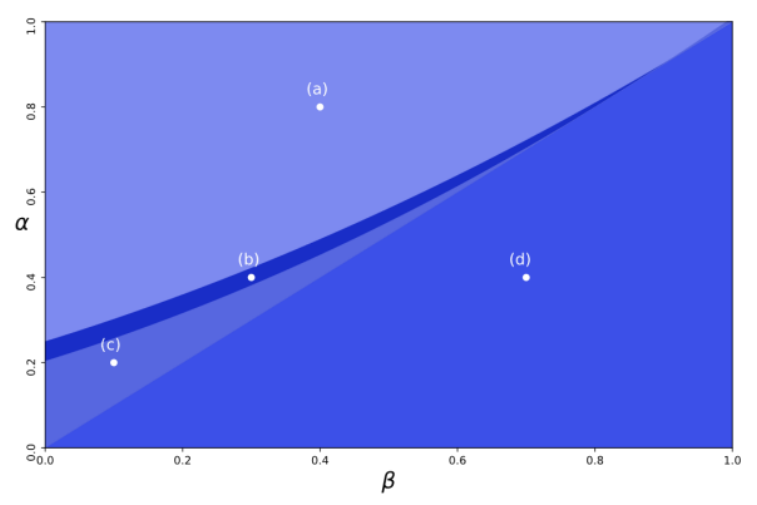

Figure 1. The existence regions of equilibrium points

\subsection{Numerical Simulations}

In this part, the numerical simulations of the system (7) are illustrated. We give some flow of solutions to demonstrate the stability around the equilibrium points that 
associate with the previous theoretical results. We apply $4^{t h}$-order Runge Kutta schemes as the numerical methods with stepsize $\Delta t=0.01$. For the sake of simulation needs, we use hypothetical parameter values due to the absence of field data.

We first set some parameter values as follows,

$$
\kappa=0.3, \quad \delta=0.4, \varepsilon=0.1, \omega=0.05
$$

By setting the parameter values as in eq. (16) and fixing $h=0.2$, we plot the existence regions of equilibrium points as in fig. 1 . Take a look at the region (a) in fig. 1. Based on the existing condition above, this circumstance is done when the Allee effect is strong. We have that all equilibrium points do not exists. Now, we choose a point in the region (b) i.e $(\alpha, \beta)=(0.4,0.3)$. We obtain two equilibrium points $\hat{E}_{1}=(0.5,0)$ and $\hat{E}_{2}=(0.2,0)$ which are also occur in a strong Allee effect. Based on Theorem 1-2, Both equilibrium points are not stable, see fig. 2.

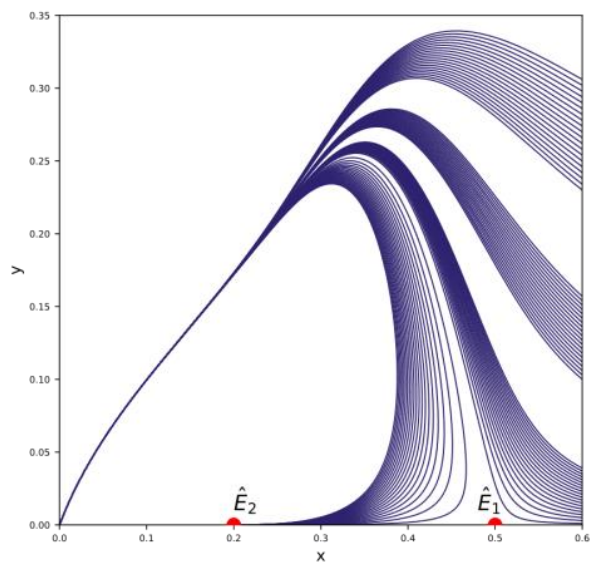

Figure 2. The flow of solutions of system (7) with parameter values as in eq. (16) and $\alpha=0.4, \beta=0.3, h=0.1$

Now, we increase $h$ to 0.5 so that $h>\delta$ is satisfied. According to Theorem $1, \hat{E}_{1}=(0.5,0)$ change its sign from a saddle point to a locally asymptotically stable point. Thus, we have a saddle point and a locally asymptotically stable point. The flow of solutions is shown in fig. 3.

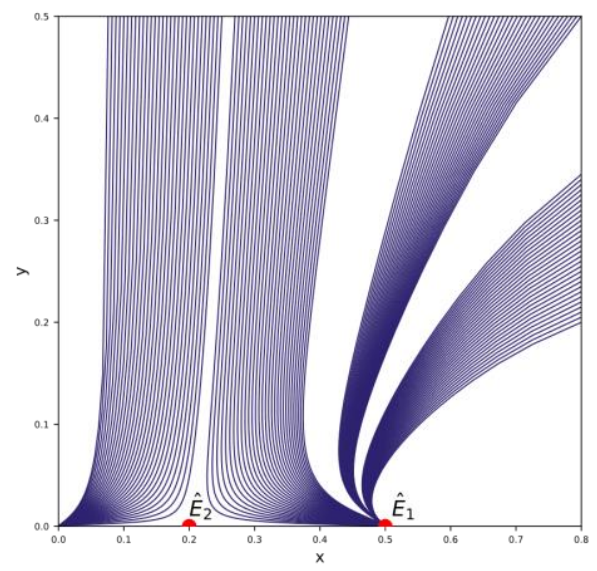

Figure 3. The flow of solutions of system (7) with parameter values as in eq. (16) and $\alpha=0.4, \beta=0.3, h=0.5$ 


\section{Panigoro et.al}

Furthermore, we replace the values of $\alpha$ and $\beta$ with a point as in in fig. 1 region (c) which is defined by $(\alpha, \beta)=(0.2,0.1)$. This circumstance also describes the dynamics of the system (7) when the Allee effect is strong. By utilizing the existing conditions along with stability conditions in Theorem 1-3, system (7) has four equilibrium points viz. three saddle points $\hat{E}_{1}=(0.7702,0), \hat{E}_{2}=(0.1298,0)$ and $E_{2}^{*}=(0.1422,0.1066) ;$ and a locally asymptotically stable $E_{1}^{*}=(0.5741,0.4306)$. The flow of solutions of this condition is shown in fig. 4 .

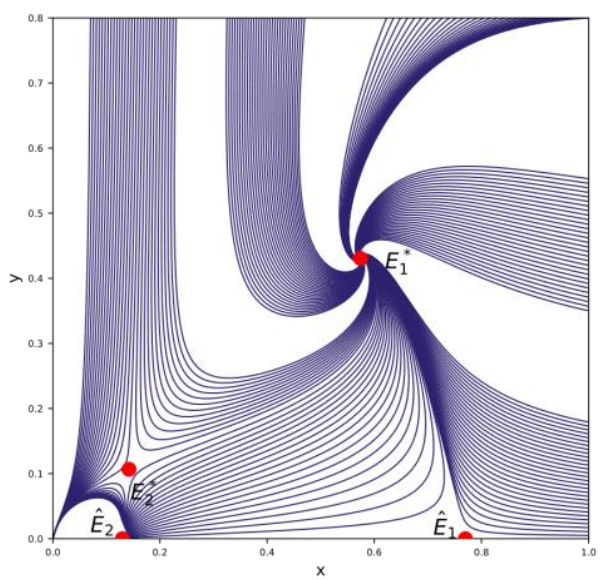

Figure 4. The flow of solutions of system (7) with parameter values as in eq. (16) and $\alpha=0.2, \beta=0.1, h=0.1$

Finally, the numerical solution of system (7) when the Allee effect is weak is demonstrated. The parameter values are set as in eq. (16) by fixing $h=0.1$. By choosing a point in fig. 1 region (d) i.e $(\alpha, \beta)=(0.4,0.7)$, we have two equilibrium points viz. a saddle point $\hat{E}_{1}=(0.7179,0)$ and a locally asymptotically stable point $E_{1}^{*}=$ $(0.5564,0.4173)$. The flow of solutions is given in fig. 5 .

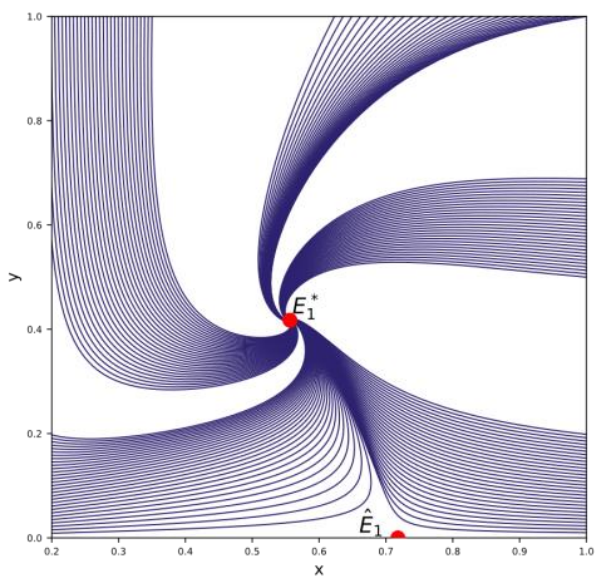

Figure 4. The flow of solutions of system (7) with parameter values as in eq. (16) and $\alpha=0.4, \beta=0.7, h=0.1$

\section{Conclusion}

We have shown the dynamics of the system (7), especially the behavior of solutions around the equilibrium points. We present the way to transform a non-autonomous system to an autonomous system. Although the system has a higher dimension, we have another effort that the dynamics become easier to investigate. We have completely 
presented that system (7) has at most four equilibrium points when the Allee effect is strong, and two equilibrium points for the weak Allee effect. The dynamics around the equilibrium point also investigated appropriately. At the end of our works, we demonstrate some phase portraits that associated to the theoretical results. One interesting issue that was not discussed in our work is dynamics around the limit-cycle $u^{2}+v^{2}=1$. We also don't examine the existence of bifurcation although the indication appears such as the change of equilibrium numbers when a parameter is varied (the occurrence of saddle-node bifurcation) and the existence of non-hyperbolic equilibrium point. Some little important works also were not discussed such as the existence, uniqueness and permanence of solution, and the global stability of equilibrium points. Hopefully, we can finish the rest of it.

\section{References}

[1] A. Suryanto, I. Darti, H. S. Panigoro, and A. Kilicman, "A Fractional-Order Predator-Prey Model with Ratio-Dependent Functional Response and Linear Harvesting," Mathematics, vol. 7, no. 11, p. 1100, Nov. 2019, DOI: $10.3390 /$ math7111100.

[2] M. Moustafa, M. H. Mohd, A. I. Ismail, and F. A. Abdullah, "Stage Structure and Refuge Effects in the Dynamical Analysis of a Fractional Order RosenzweigMacArthur Prey-Predator Model," Prog. Fract. Differ. Appl., vol. 5, no. 1, pp. 4964, 2019, DOI: 10.18576/pfda/050106.

[3] J. Ghosh, B. Sahoo, and S. Poria, "Prey-Predator Dynamics with Prey Refuge Providing Additional Food to Predator," Chaos, Solitons and Fractals, vol. 96, pp. 110-119, 2017, DOI: 10.1016/j.chaos.2017.01.010.

[4] N. Hasan, R. Resmawan, and E. Rahmi, "Analisis Kestabilan Model EkoEpidemiologi dengan Pemanenan Konstan pada Predator," J. Mat. Stat. dan Komputasi, vol. 16, no. 2, p. 121, Dec. 2019, DOI: 10.20956/jmsk.v16i2.7317.

[5] H. S. Panigoro, A. Suryanto, W. M. Kusumawinahyu, and I. Darti, “Dynamics of a Fractional-Order Predator-Prey Model with Infectious Diseases in Prey," Commun. Biomath. Sci., vol. 2, no. 2, pp. 105-117, 2019, DOI: 10.5614/cbms.2019.2.2.4.

[6] P. H. Leslie and J. C. Gower, "The Properties of a Stochastic Model for the Predator-Prey Type of Interaction Between Two Species," Biometrika, vol. 47, no. 3\&4, pp. 219-234, 1960, DOI: 10.2307/2333294.

[7] A. Suryanto, I. Darti, and S. Anam, "Stability Analysis of a Fractional Order Modified Leslie-Gower Model with Additive Allee Effect," Int. J. Math. Math. Sci., vol. 2017, no. 11, pp. 1-9, 2017, DOI: 10.1155/2017/8273430.

[8] P. C. Tobin, L. Berec, and A. M. Liebhold, "Exploiting Allee Effects for Managing Biological Invasions," Ecol. Lett., vol. 14, no. 6, pp. 615-624, 2011, DOI: 10.1111/j.1461-0248.2011.01614.x.

[9] E. Rahmi and H. S. Panigoro, "Pengaruh Pemanenan terhadap Model Verhulst dengan Efek Allee," in SEMIRATA MIPAnet 2017, no. 1, 2017, pp. 105-112.

[10] Y. Ye, H. Liu, Y. Wei, K. Zhang, M. Ma, and J. Ye, “Dynamic Study of a PredatorPrey Model with Allee Effect and Holling Type-I Functional Response," Adv. 


\section{Panigoro et.al}

Differ. Equations, vol. 2019, no. 1, pp. 1-15, 2019, DOI: 10.1186/s13662-019-2311-1.

[11] Y. Cai, C. Zhao, W. Wang, and J. Wang, "Dynamics of a Leslie-Gower PredatorPrey model With Additive Allee Effect," Appl. Math. Model., vol. 39, no. 7, pp. 2092-2106, 2015, DOI: 10.1016/j.apm.2014.09.038.

[12] Y. Liu, Z. Liu, and R. Wang, "Bogdanov-Takens Bifurcation with Codimension Three of a Predator-Prey System Suffering the Additive Allee Effect," Int. J. Biomath., vol. 10, no. 3, pp. 1-24, 2017, DOI: 10.1142/S1793524517500449.

[13] X. Liu, G. Fan, and T. Zhang, "Evolutionary Dynamics of Single Species Model with Allee Effect," Phys. A Stat. Mech. its Appl., vol. 526, 2019, DOI: 10.1016/j.physa.2019.04.010.

[14] P. Feng and Y. Kang, "Dynamics of a Modified Leslie-Gower Model with Double Allee Effects," Nonlinear Dyn., vol. 80, no. 1-2, pp. 1051-1062, 2015, DOI: 10.1007/s11071-015-1927-2.

[15] P. J. Pal and T. Saha, "Qualitative Analysis of a Predator-Prey System with Double Allee Effect in Prey," Chaos, Solitons and Fractals, vol. 73, pp. 36-63, 2015, DOI: 10.1016/j.chaos.2014.12.007.

[16] B. Dennis, "Allee Effects: Population Growth, Critical Density, and the Chance of Extinction," Nat. Resour. Model., vol. 3, no. 4, pp. 481-538, 1989, DOI: 10.1111/j.1939-7445.1989.tb00119.x.

[17] P. A. Stephens and W. J. Sutherland, "Consequences of the Allee Effect for Behavior, Ecology and Conservation," Trends Ecol. Evol., vol. 14, no. 10, pp. 401405, 1999, DOI: 10.1016/S0169-5347(99)01684-5.

[18] P. Aguirre, E. González-Olivares, and E. Sáez, "Two Limit Cycles in a LeslieGower Predator-Prey Model with Additive Allee Effect," Nonlinear Anal. Real World Appl., vol. 10, no. 3, pp. 1401-1416, 2009, DOI: 10.1016/j.nonrwa.2008.01.022.

[19] P. Aguirre, E. Gonzalez-Olivares, and E. Saez, "Three Limit Cycles in a LeslieGower Predator-Prey Model with Additive Allee Effect," SIAM J. Appl. Math., vol. 69, no. 5, pp. 1244-1262, 2009, DOI: 10.1137/090750688.

[20] H. S. Panigoro and E. Rahmi, “Modifikasi Sistem Predator-Prey: Dinamika Model Leslie-Gower dengan Daya Dukung yang Tumbuh Logistik," in SEMIRATA MIPAnet, 2017, pp. 94-103. 